\title{
Identification of Enterocytozoon bieneusi in goats and cattle in Thailand
}

\author{
Ruenruetai Udonsom', Rapeepun Prasertbun', Aongart Mahittikorn', Rachatawan Chiabchalard', \\ Chantira Sutthikornchai ${ }^{1}$, Attakorn Palasuwan ${ }^{2}$ and Supaluk Popruk ${ }^{1 *}$
}

\begin{abstract}
Background: Enterocytozoon bieneusi has been increasingly reported to infect domestic animals and humans, with human infections primarily reported as zoonotic in origin. The aim of the present study was to determine the presence and genotype of E. bieneusi in humans and domestic animals in central Thailand by testing stool samples of 200 apparently healthy humans, 73 goats, 60 cattle and 65 pigs using nested-PCR/ sequence analysis based on the ITS region of SSU rRNA genes.

Results: E. bieneusi tested positive in 2 (1\%) of the 200 stool samples collected from humans and 56 (28.3\%) of the 198 stool samples collected from domestic animals. The highest prevalence of E. bieneusi was observed in pigs (39/ $65,60 \%)$, followed by goats $(14 / 73,19.2 \%)$ and cattle $(3 / 60,5 \%)$. Seven novel E. bieneusi genotypes were identified, which were named GoatAYE1-4 and PigAYE1-3 and clustered in either zoonotic Group 1 or Group 2. Moreover, eleven previously described E. bieneusi genotypes were also identified (O, D, H, SX1, CHC8, CHG3, CS-10, SHZC1, LW1, WildBoar5, and EbpC). All novel genotypes exhibited zoonotic potential from a phylogenetic analysis of ITS region.
\end{abstract}

Conclusion: Our data showed that the prevalence of E. bieneusi is low in apparently healthy individuals and higher in pigs than cattle and goats. This study provides baseline data useful for controlling and preventing $E$. bieneusi infection in farm communities, where pigs and goats appear to be the major reservoir of E. bieneusi. The results of our study support the view that E. bieneusi is a zoonotic pathogen that should be considered a potential public health threat.

Keywords: Enterocytozoon bieneusi, Goats, Cattle, Pigs, Zoonotic

\section{Background}

Enterocytozoon bieneusi, a complex intestinal microsporidian pathogen with multiple genotypes, parasitises a variety of animals $[1,2]$ and causes infection both immunocompromised and immunocompetent humans including children, travellers and the elderly [3-5]. The clinical presentation of $E$. bieneusi infection ranges from being asymptomatic to presenting symptoms of dehydration, malabsorption and chronic diarrhoea [2]. In human, potential modes of $E$. bieneusi transmission are faecal-oral route and zoonotic [6-8]. Risk factors associated with $E$. bieneusi infection include impaired immunity, poor personal hygiene, deficient community

\footnotetext{
* Correspondence: supaluk.pop@mahidol.ac.th

${ }^{1}$ Department of Protozoology, Faculty of Tropical Medicine, Mahidol

University, Ratchawithi Road, Ratchathewi, Bangkok 10400, Thailand

Full list of author information is available at the end of the article
}

sanitation and close contact with infected animals or humans [2].

Routine laboratory diagnosis of microsporidian infection involves staining and light microscopy. However, identification of this pathogen is challenging because of the small size of its spores [9], leading to underestimating of E. bieneusi prevalence. Polymerase chain reaction (PCR) is the most powerful, sensitive and specific technique for detecting and genotyping E. bieneusi. Based on $\mathrm{PCR} /$ sequence analysis of the internal transcribed spacer (ITS) region of small-subunit ribosomal RNA (SSU rRNA) genes, >500 genotypes and 11 phylogenetic groups of E. bieneusi have been described in humans and animals $[2,10,11]$. E. bieneusi genotypes in Group 1 and Group 2 may be zoonotic genotypes responsible for most human infections $[10,12]$. In animals, several studies indicated that pigs may be the major reservoir of $E$.

(c) The Author(s). 2019 Open Access This article is distributed under the terms of the Creative Commons Attribution 4.0 International License (http://creativecommons.org/licenses/by/4.0/), which permits unrestricted use, distribution, and 
bieneusi and a potential source of $E$. bieneusi infection in humans $[8,13-15]$. Since there is limited information on the E. bieneusi genotypes in apparently healthy individuals in Thailand, most available information was studied in orphans and HIV-patients and previously studied target animals were only cats and pigs. We conducted this study to identify the prevalence and genotypes of E. bieneusi circulating among apparently healthy individuals and domestic animals especially, goats and cattle in central Thailand.

\section{Results}

The prevalence of E. bieneusi in animals and humans was 28.3 and 1\%, respectively (Table 1). The highest prevalence of $E$. bieneusi occurs in pigs (60\%), followed by goats (19.2\%) and cattle (5\%). Genotype analysis using the ITS region sequence in the 58 positive samples detected 18 different genotypes. These genotypes included 11 that are known [D, O, H, SX1, CHC8, CS-10, CHG3, LW1, EbpC, WildBoar5, and SHZC1] and seven genotypes that are novel [GoatAYE1-4 and PigAYE1-3]. The predominant genotypes of E. bieneusi identified in goats, pigs and cattle were $\mathrm{CHC} 8$ / CHG3, EbpC and D, respectively. Our phylogenetic analysis revealed that the five novel genotypes were clustered in zoonotic Group1 (PigAYE1-3 and GoatAYE1-2), whereas the others were in zoonotic Group2 (GoatAYE3-4) (Fig. 1).

Table 2 shows nucleotide differences between the seven novel genotypes and previously identified genotypes. The novel genotypes GoatAYE1 and GoatAYE2 showed one nucleotide difference in the ITS region relative to genotype $\mathrm{H}$ at position $245 \mathrm{C} \rightarrow \mathrm{T}$ and position $126 \mathrm{~A} \rightarrow \mathrm{G}$, respectively. The novel genotype GoatAYE3 showed two nucleotide differences in the ITS region relative to genotype $\mathrm{CHC} 8$ (position $95 \mathrm{~T} \rightarrow \mathrm{C}$ and $163 \mathrm{G} \rightarrow \mathrm{A}$ ), whereas GoatAYE4 showed one nucleotide difference from genotype CHG3 (position $134 \mathrm{G} \rightarrow \mathrm{A}$ ). Moreover, the novel genotype PigAYE1 showed one nucleotide difference from genotype CS-10 (position $21 \mathrm{G} \rightarrow \mathrm{A}$ ), while PigAYE3 showed two nucleotides difference relative to CS-10 (position $21 \mathrm{G} \rightarrow \mathrm{A}$ and position $246 \mathrm{~A} \rightarrow \mathrm{C}$ ). In addition, PigAYE2 showed one nucleotide difference to genotype PigEBITS5 (position $131 \mathrm{~T} \rightarrow \mathrm{C}$ ).

\section{Discussion}

In this study, the overall prevalence of $E$. bieneusi in humans and animals was $14.6 \%$. The highest prevalence

Table 1 Prevalence and genotypes of Enterocytozoon bieneusi in Ayutthaya Province, Thailand

\begin{tabular}{|c|c|c|}
\hline Host & Prevalence (\%) & Genotype (synonyms)/ Positive count (GenBank accession no.) \\
\hline \multirow[t]{2}{*}{ Humans } & $2 / 200(1)$ & O/ 1 (KU245704) \\
\hline & & D (WL8, Peru9, PigEBITS9, PtEb VI, CEbc)/ 1 (MK168302) \\
\hline \multicolumn{3}{|l|}{ Animals } \\
\hline \multirow[t]{8}{*}{ Goats } & $14 / 73(19.2)$ & GoatAYE1 $/ 1$ \\
\hline & & GoatAYE2 $/ 1$ \\
\hline & & GoatAYE3a $/ 1$ \\
\hline & & GoatAYE4 ${ }^{\mathrm{a}} / 1$ \\
\hline & & H (PEbC) / 1 (KP318000) \\
\hline & & SX1/ 1 (KT235712) \\
\hline & & CHC8/ 4 (MK573332) \\
\hline & & $\mathrm{CHG} 3 / 4(\mathrm{MH} 822618)$ \\
\hline \multirow[t]{9}{*}{ Pigs } & $39 / 65(60)$ & CS-10/ 1 (KP259313) \\
\hline & & SHZC1/1 (MG183830) \\
\hline & & PigAYE1 ${ }^{a} / 2$ \\
\hline & & PigAYE2a / 1 \\
\hline & & PigAYE3 / 1 \\
\hline & & $\mathrm{H}(\mathrm{PEbC}) / 2$ (KP318000) \\
\hline & & LW1 (Henan-I) / 6 (KX008325) \\
\hline & & WildBoar5/ 9 (KF383402) \\
\hline & & Ebpc (E,Peru4,WL13,WL17)/ 16 (MH024028) \\
\hline Cattle & $3 / 60(5)$ & D (WL8, Peru9, PigEBITS9, PtEb VI, CEbc) / 3 (MK168302) \\
\hline Total & $56 / 198(28.3)$ & \\
\hline Total & $58 / 398(14.6)$ & \\
\hline
\end{tabular}

${ }^{\mathrm{a}}$ Novel genotypes found in this study 


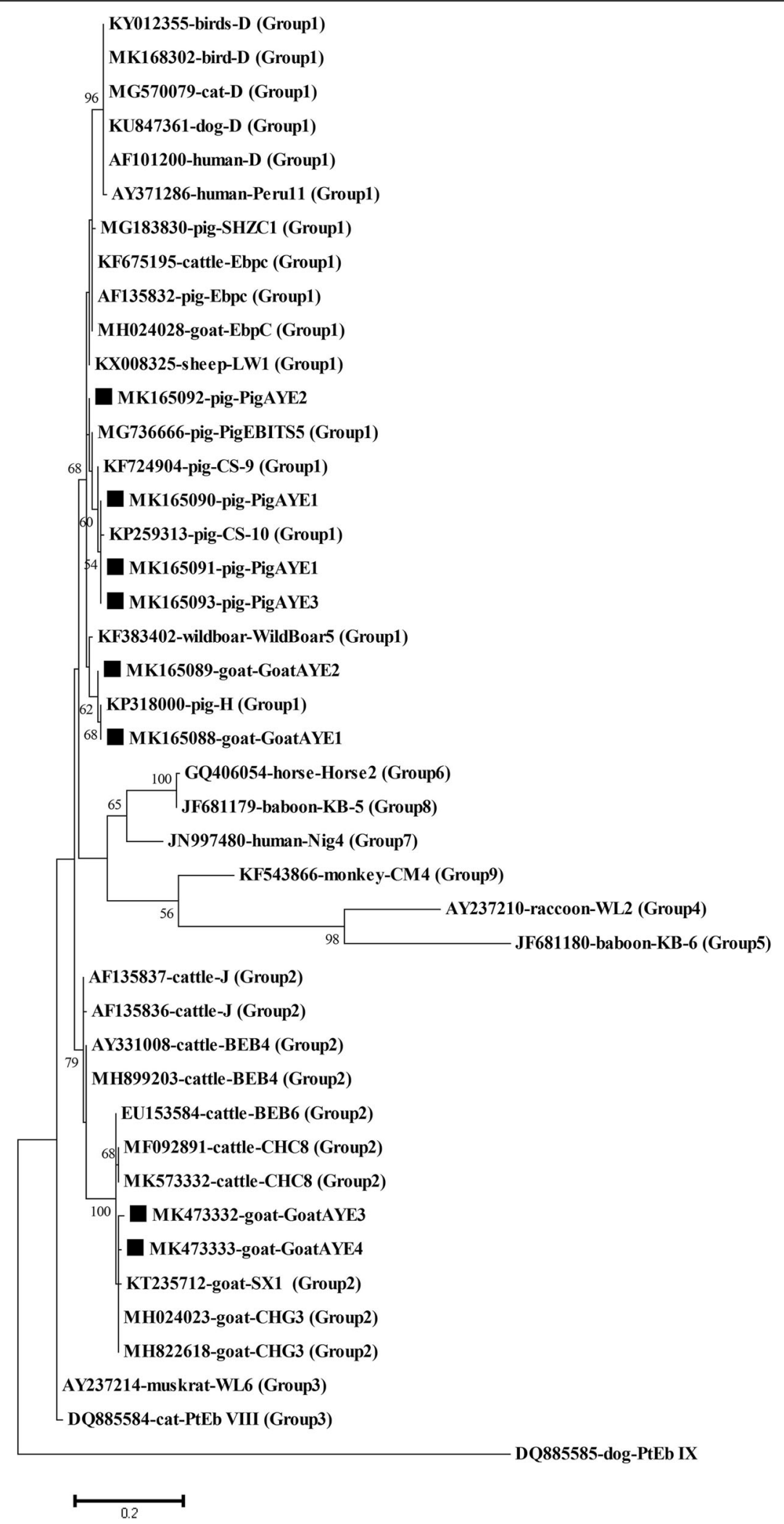

Fig. 1 Phylogenetic tree of E. bieneusi isolates and reference for the internal transcribed spacer (ITS) region sequence of small-subunit ribosomal RNA (SSU rRNA) genes obtained from GenBank. The outgroup sequence was the dog-specific E. bieneusi genotype. Values on nodes represent bootstrap support using maximum likelihood methods. Symboln = novel genotypes identified in this study 
Table 2 Variations in ITS region sequences of SSU rRNA genes of Enterocytozoon bieneusi isolates identified in this study and comparison with three known genotypes

\begin{tabular}{|c|c|c|c|c|c|c|c|c|c|c|}
\hline \multicolumn{2}{|c|}{ Genotypes (no.) } & \multicolumn{8}{|c|}{ Nucleotide at position } & \multirow{3}{*}{$\begin{array}{l}\text { GenBank accession } \\
\text { no. } \\
\text { MK165088 }\end{array}$} \\
\hline & & \multirow{2}{*}{21} & \multirow{2}{*}{$\frac{95}{-}$} & \multirow{2}{*}{$\begin{array}{l}126 \\
-\end{array}$} & \multirow{2}{*}{$\frac{131}{-}$} & \multirow{2}{*}{$\frac{134}{-}$} & \multirow{2}{*}{$\frac{163}{-}$} & \multirow{2}{*}{$\frac{245}{T}$} & \multirow{2}{*}{$\frac{246}{-}$} & \\
\hline Novel & GoatAYE1 & & & & & & & & & \\
\hline & GoatAYE2 & - & - & G & - & - & - & - & - & MK165089 \\
\hline Known & $\mathrm{H}$ & - & - & A & - & - & - & $C$ & - & KP318000 \\
\hline Novel & GoatAYE3 & - & $C$ & - & - & - & A & - & - & MK473332 \\
\hline Known & $\mathrm{CHC8}$ & - & $\mathrm{T}$ & - & - & - & G & - & - & MK573332 \\
\hline Novel & GoatAYE4 & - & - & - & - & A & - & - & - & MK473333 \\
\hline Known & $\mathrm{CHG} 3$ & - & - & - & - & G & - & - & - & MH822618 \\
\hline \multirow[t]{2}{*}{ Novel } & PigAYE1 & A & - & - & - & - & - & - & - & MK165090/ MK165091 \\
\hline & PigAYE3 & A & - & - & - & - & - & - & C & MK165093 \\
\hline Known & CS-10 & G & - & - & - & - & - & - & A & KP259313 \\
\hline Novel & PigAYE2 & - & - & - & $C$ & - & - & - & - & MK165092 \\
\hline Known & PigEBITS5 & - & - & - & $\mathrm{T}$ & - & - & - & - & MG736666 \\
\hline
\end{tabular}

of $E$. bieneusi in animals occurs in pigs $(60 \%)$, followed by goats $(19.2 \%)$ and cattle $(5 \%)$, whereas the prevalence of $E$. bieneusi in humans was detected in only $1 \%$. Other studies in Thailand found that the prevalence of $E$. bieneusi in humans ranged from $1.3-27.27 \%$ and varied from country to country $[8,16-20]$. Divergences in the prevalence of infection might due to differences in the immunity of hosts when stool samples were collected, personal hygiene, cultural norms, and detection methods. The prevalence of $E$. bieneusi infection in pigs observed in our study was higher than prevalence reported by other studies in Thailand and elsewhere, such as in China and Central Europe (5.4$28.1 \%)[8,17,21,22]$, but similar to a study reported by Fiuza et al. in pigs in Brazil (59.3\%) [14]. There are only a few studies reported a higher prevalence of $E$. bieneusi in pigs than our study (83.2-94\%) [23, 24]. We also found that there was a higher prevalence of $E$. bieneusi in pigs aged 24 months than other ages, which is consistent with studies on pigs from Brazil, China, and Thailand [14, 17, 25, 26]. The higher prevalence among piglets may due to lower immunity and higher stress [27]. Moreover, cages with a high density of pigs may cause more opportunities for transmitting $E$. bieneusi infection among animals than less denselypacked cages. To the best of our knowledge, this is the first study to report the prevalence of E. bieneusi in cattle (5\%) and goats (19.2\%) in Thailand. The low prevalence of $E$. bieneusi in cattle found in Thailand contradicts the results of studies conducted in Argentina, Brazil, China, and South Korea [28-32]. The prevalence of $E$. bieneusi in goats was also lower than the prevalence rates found in previous studies from China and Egypt (20.5-28.8\%) [33-36], except for the studies by Li et al. and Lores et al. (4.1-14.2\%) [37, 38]. These diversities between studies in prevalence among animals might be related to the age and/or immunity of sampled animals, as well as to geographical differences. In our study, most of the tested cattle and goats were older than six months, so the experimental animals may have already developed immunity against $E$. bieneusi.

In this study, we detected 11 previously described and seven novel genotypes of E. bieneusi in human and animal samples. In Thailand, previous studies have determined that genotype $\mathrm{D}$ is commonly observed in both HIV-positive patients, healthy individuals and animals, such as in Indian peafowl and calf [8, 19, 28, 39-42]. This genotype was found from all $E$. bieneusi-positive cattle in this study.

Genotype $\mathrm{O}$ has been observed in humans, pigs, cattle and dogs in previous studies conducted in various countries worldwide [8, 14, 19, 24, 30, 43]. Nine of 18 E. bieneusi genotypes identified in our study occurred in pigs with the predominant genotype consisting of EbpC, followed by LW1 and $\mathrm{H}$. In Thailand, genotypes EbpC and $H$ have been reported in humans and pigs $[8,17,19]$, which is consistent with previous studies conducted in other countries $[14,24,44,45]$. Genotype EbpC has also been identified in cattle, dogs, goats, and monkeys [28, 43, 46, 47], suggesting that this genotype is not host-specific. Although genotype LW1 was reported for the first time from pigs in Thailand, this genotype has been previously identified in humans, pigs and wild boars in Australia and China [22, 24, 25, 45]. Surprisingly, five of $39 \mathrm{E}$. bieneusi-infected pigs we genotyped as WildBoar5, a genotype that has also been identified from wild boars in Central Europe [22]. Due to the fact that pig and wild boar belong to the same species, they are likely parasitised by the same species [22]. Moreover, genotypes CS-10 and SHZC1 in pigs, genotypes SX1, CHC8 and $\mathrm{CHG} 3$ in goats have also been identified and 
consistent with previous studies [26, 27], except that CHC8 has been previously identified in cattle [29, 34, 47]. Phylogenetic analyses provided further information that the five novel genotypes were clustered in zoonotic Group1 (PigAYE1-3 and GoatAYE1-2) and the others were grouped in zoonotic Group 2 (GoatAYE3-4), which implies that they have tremendous potential to be zoonotic. Apparently, genetic diversity definitively exists in this pathogen and zoonotic potential of these novel genotypes should be characterised further in future studies.

\section{Conclusions}

Our results revealed a high prevalence of E. bieneusi in pigs $(60 \%)$ and identified a high number of zoonotic genotypes, while goats and cattle were also carriers of $E$. bieneusi. Therefore, it is important to control E. bieneusi-infected animals on farms by properly managing animal waste. To better understand the molecular epidemiology and zoonotic potential of E. bieneusi, it will be necessary to increase sample size, examine a wider variety of animal species and expand the survey area.

\section{Methods}

\section{Specimen collection}

A cross-sectional study of $E$. bieneusi infection was conducted from five Thai sub-districts (Khanon Luang, Talat Kriap, Ban Pho, Wat Yom, and Ko Koet) in the Bang Pa-In District Ayutthaya Province, central Thailand. In total, 200 apparently healthy individual stool samples and 198 stool samples from domestic animals, including 73 goats (Farms 1-3), 60 cattle (Farms 4-6) and 65 pigs (Farms 7-9) and human participants aged from 1 to 80 years (Fig. 2). No participants complained about any gastrointestinal symptoms, such as abdominal pain or diarrhoea throughout the experiment. All nine farms participated in our study were small and private farms. Ages of goats on Farms 1-3 ranged between 4 and 12 months, ages of cattle on Farms 4-6 were more than 6 months, while ages of pigs on Farms 7,8 and 9 ranged between 2 and 5 months, 3-6 months and 3-8 months, respectively. Types of animals were selected based primarily on their proximity to humans in experimental areas. Fresh stool samples were collected either directly from animals' rectum; each animal was

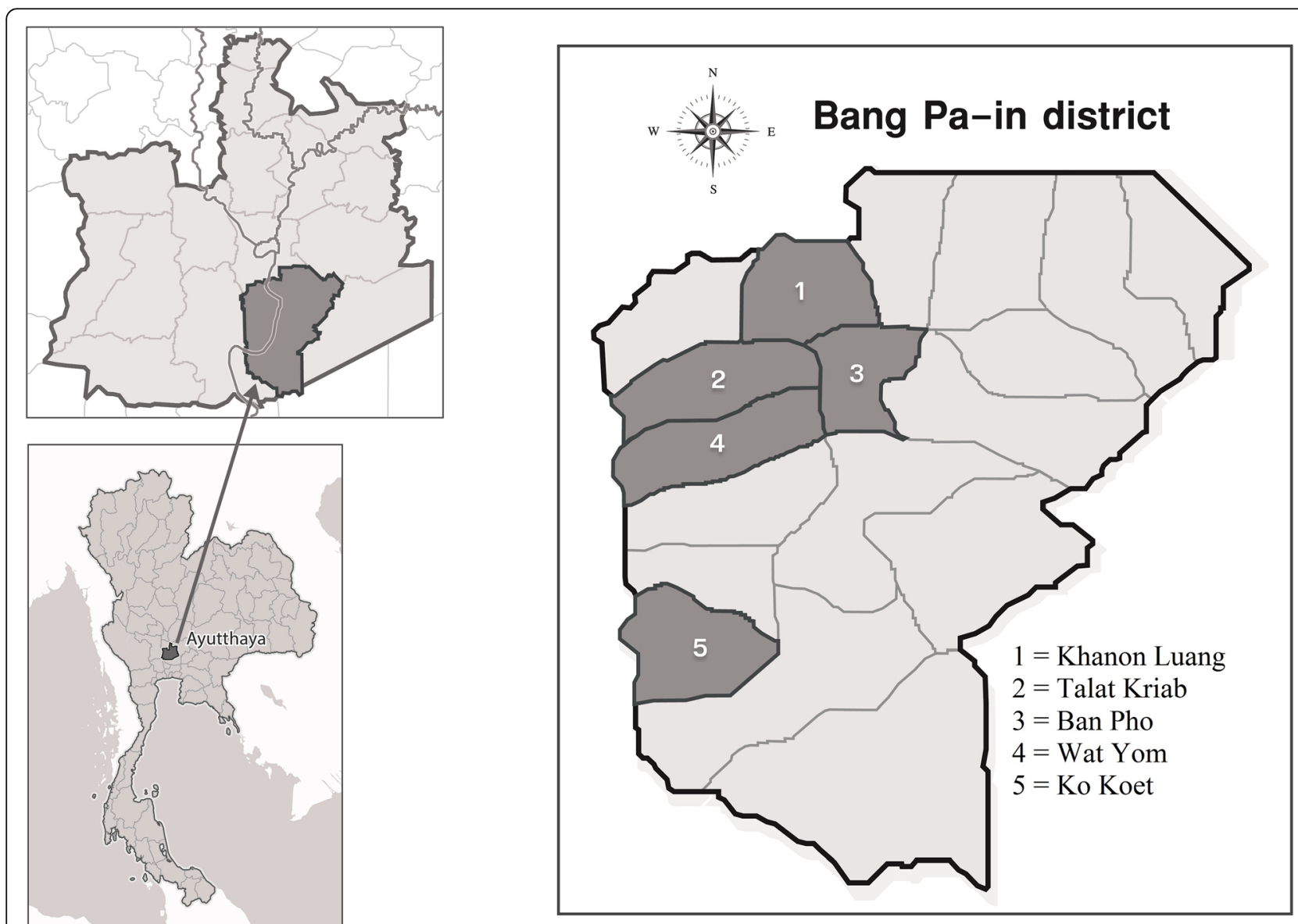

Fig. 2 Map of study areas in Bang Pa-In District, Ayutthaya Province, central Thailand (Design by miss Chompunuch Sangpan (our co-worker in Faculty of Tropical Medicine)) 
securely detained in an individual cage, or immediately after defecation (each sample was separately taken from the middle of the stool with a clean plastic spoon).

All stool samples were safely stored under cool conditions during transportation to the Department of Protozoology, Faculty of Tropical Medicine, Mahidol University and preserved at $-20^{\circ} \mathrm{C}$ before extracting DNA.

\section{DNA extraction and nested PCR}

DNA was extracted from stool samples using a commercially available DNA extraction kit (PSP Spin Stool Kit, Stratec Molecular, Berlin, Germany) following the manufacturer's instructions. We amplified a fragment of the ITS region of SSU rRNA genes from the extracted DNA using nested PCR. The amplicons were about 390 base pairs (bps) in length [13]. The outer primer set was EBITS3 (5' - GGT CAT AGG GAT GAA GAG - 3') and EBITS4 (5'- TTC GAG TTC TTT CGC GCT C-3'), whereas the inner primer set was EBITS1 (5'- GCT CTG AAT ATC TAT GGC T-3') and EBITS2.4 (5'ATC GCC GAC GGA TCA AGT G-3'). The thermal cycling conditions were maintained as follows: initial denaturation at $94^{\circ} \mathrm{C}$ for $3 \mathrm{~min}, 35$ cycles of denaturation at $94{ }^{\circ} \mathrm{C}$ for $30 \mathrm{~s}$, annealing at $55^{\circ} \mathrm{C}$ for $30 \mathrm{~s}$, extension at $72{ }^{\circ} \mathrm{C}$ for $40 \mathrm{~s}$, followed by final extension at $72^{\circ} \mathrm{C}$ for $10 \mathrm{~min}$. The final PCR products (390 bps) were separated on $2 \%$ agarose gel and visualised under a UV transilluminator.

\section{Sequencing and phylogenetic analysis}

All positive PCR products from the second PCR reaction were purified before sequencing with an ABI 3730xl DNA analyser using the BigDye Terminator Cycle Sequencing kit (Applied Biosystems). All sequences of E. bieneusi-positive samples were checked for similarity with previously published sequences stored in the GenBank database using BLAST (https://blast.ncbi.nlm.nih.gov/Blast.cgi). The nucleotide sequences of novel $E$. bieneusi isolates were submitted to GenBank under accession numbers MK165088MK165093 and MK473332-MK473333.

The sequences of $E$. bieneusi-positive samples and an outgroup sequence (GenBank accession no. DQ885585) were aligned using Clustal W and phylogenetic analysis was performed with MEGA version 6 software (http:// www.megasoftware.net) [48]. The Tamura-Nei model was used to account for the evolution of the DNA sequences and constructed a phylogenetic tree using maximum likelihood methods with 1000 bootstrap replicates.

\section{Statistical analysis}

Descriptive analyses (percentages) were used to describe the prevalence of positive stool samples and the distributions of $E$. bieneusi genotypes throughout the study region.

\section{Abbreviations}

bps: base pairs; ITS: Internal transcribed spacer; PCR: Polymerase chain reaction; SSU rRNA: Small-subunit ribosomal RNA

\section{Acknowledgments}

We thank Enago for editing this manuscript and Miss Chompunuch Sangpan (our co-worker in Faculty of Tropical Medicine) for providing the graphical design of Fig. 2.

Authors' contributions

$R U, R P, A M, R C, C S, A P$, and SP conceived experiments. RP, AM, RC, CS, and AP collected samples from the field. RU and SP performed laboratory experiments. RU, AP, and SP analysed and interpreted data. RU and SP wrote the manuscript. SP critically revised the manuscript. All authors contributed to, read and approved the final version of the manuscript.

\section{Funding}

This manuscript was supported by the Faculty of Tropical Medicine, Mahidol University, Thailand for English editing and article-processing charges, but did not have any additional roles in the data collection or analyses pertaining to this manuscript.

\section{Availability of data and materials}

The data sets used and/or analysed during the present study are available from the corresponding author by reasonable request.

\section{Ethics approval and consent to participate}

Ethics approval for experiments using human samples was obtained from the Ethics Review Committee for Research Involving Human Research Subjects, Health Sciences Group, Chulalongkorn University (COA no. 152/ 2558). The objectives, protocols and potential risks were clearly explained to all participants. Written informed consent was obtained from all participants. Parents/guardians provided consent on behalf of children.

For animal studies, ethical clearance was obtained from the Ethics

Committee at the Faculty of Tropical Medicine-Animal Care and Use Committee, Mahidol University (FTM-ACUC 013/2018E). The objectives, procedures and potential risks were verbally explained to each animal owner from which we obtained written informed consent.

Consent for publication

Not applicable

\section{Competing interests}

The authors declare that they have no competing interests.

\section{Author details}

'Department of Protozoology, Faculty of Tropical Medicine, Mahidol University, Ratchawithi Road, Ratchathewi, Bangkok 10400, Thailand. ${ }^{2}$ Oxidation in Red Cell Disorders and Health Task Force, Department of Clinical Microscopy, Faculty of Allied Health Sciences, Chulalongkorn University, 154 Rama I Road, Bangkok 10330, Thailand.

Received: 3 June 2019 Accepted: 19 August 2019

Published online: 28 August 2019

References

1. Wang SS, Wang RJ, Fan XC, Liu TL, Zhang LX, Zhao GH. Prevalence and genotypes of Enterocytozoon bieneusi in China. Acta Trop. 2018;183:142-52.

2. Matos O, Lobo ML, Xiao L. Epidemiology of Enterocytozoon bieneusi infection in humans. J Parasitol Res. 2012;2012:981424.

3. Lores B, Lopez-Miragaya I, Arias C, Fenoy S, Torres J, del Aguila C. Intestinal microsporidiosis due to Enterocytozoon bieneusi in elderly human immunodeficiency virus-negative patients from Vigo. Spain Clin Infect Dis. 2002;34:918-21.

4. Mungthin M, Subrungruang I, Naaglor T, Aimpun P, Areekul W, Leelayoova S. Spore shedding pattern of Enterocytozoon bieneusi in asymptomatic children. J Med Microbiol. 2005:54:473-6.

5. Wichro E, Hoelzl D, Krause R, Bertha G, Reinthaler F, Wenisch C. Microsporidiosis in travel-associated chronic diarrhea in immune-competent patients. Am J Trop Med Hyg. 2005;73:285-7. 
6. Cheng HW, Lucy FE, Graczyk TK, Broaders MA, Mastitsky SE. Municipal wastewater treatment plants as removal systems and environmental sources of human-virulent microsporidian spores. Parasitol Res. 2011;109: 595-603.

7. Decraene V, Lebbad M, Botero-Kleiven S, Gustavsson AM, Lofdahl M. First reported foodborne outbreak associated with microsporidia, Sweden, October 2009. Epidemiol Infect. 2012;140:519-27.

8. Prasertbun R, Mori H, Pintong AR, Sanyanusin S, Popruk S, Komalamisra C, et al. Zoonotic potential of Enterocytozoon genotypes in humans and pigs in Thailand. Vet Parasitol. 2017:233:73-9.

9. Rinder H, Thomschke A, Dengjel B, Gothe R, Loscher T, Zahler M. Close genotypic relationship between Enterocytozoon bieneusi from humans and pigs and first detection in cattle. J Parasitol. 2000;86:185-8.

10. Li W, Feng Y, Santin M. Host specificity of Enterocytozoon bieneusi and public health implications. Trends Parasitol. 2019;35:436-51.

11. Li W, Wan Q, Yu Q, Yang Y, Tao W, Jiang Y, et al. Genetic variation of miniand microsatellites and a clonal structure in Enterocytozoon bieneusi population in foxes and raccoon dogs and population differentiation of the parasite between fur animals and humans. Parasitol Res. 2016:115:2899-904.

12. Xu C, Ma X, Zhang H, Zhang XX, Zhao JP, Ba HX, et al. Prevalence, risk factors and molecular characterization of Enterocytozoon bieneusi in raccoon dogs (Nyctereutes procyonoides) in five provinces of northern China. Acta Trop. 2016;161:68-72.

13. Buckholt MA, Lee JH, Tzipori S. Prevalence of Enterocytozoon bieneusi in swine: an 18-month survey at a slaughterhouse in Massachusetts. Appl Environ Microbiol. 2002;68:2595-9.

14. Fiuza VR, Oliveira FC, Fayer R, Santin M. First report of Enterocytozoon bieneusi in pigs in Brazil. Parasitol Int. 2015;64:18-23.

15. Dengjel B, Zahler M, Hermanns W, Heinritzi K, Spillmann T, Thomschke A, et al. Zoonotic potential of Enterocytozoon bieneusi. J Clin Microbiol. 2001;39: 4495-9

16. Pagornrat W, Leelayoova S, Rangsin R, Tan-Ariya P, Naaglor T, Mungthin M. Carriage rate of Enterocytozoon bieneusi in an orphanage in Bangkok, Thailand. J Clin Microbiol. 2009;47(11):3739-41.

17. Leelayoova S, Piyaraj P, Subrungruang I, Pagornrat W, Naaglor T, Phumklan $\mathrm{S}$, et al. Genotypic characterization of Enterocytozoon bieneusi in specimens from pigs and humans in a pig farm community in Central Thailand. J Clin Microbiol. 2009:47:1572-4.

18. Leelayoova S, Subrungruang I, Rangsin R, Chavalitshewinkoon-Petmitr P, Worapong J, Naaglor T, et al. Transmission of Enterocytozoon bieneusi genotype a in a Thai orphanage. Am J Trop Med Hyg. 2005;73:104-7.

19. Leelayoova S, Subrungruang I, Suputtamongkol Y, Worapong J, Petmitr PC, Mungthin M. Identification of genotypes of Enterocytozoon bieneusi from stool samples from human immunodeficiency virus-infected patients in Thailand. J Clin Microbiol. 2006:44:3001-4.

20. Wanachiwanawin D, Manatsathit S, Lertlaituan P, Thakerngpol K, Suwanagool P. Intestinal microsporidiosis in HIV-infected patients with chronic diarrhea in Thailand. Southeast Asian J Trop Med Public Health. 1998:29:767-71.

21. Zhang $X$, Wang $Z$, Su $Y$, Liang $X$, Sun $X$, Peng $S$, et al. Identification and genotyping of Enterocytozoon bieneusi in China. J Clin Microbiol. 2011;49: 2006-8

22. Nemejc K, Sak B, Kvetonova D, Hanzal V, Janiszewski P, Forejtek $P$, et al. Prevalence and diversity of Encephalitozoon spp. and Enterocytozoon bieneusi in wild boars (Sus scrofa) in Central Europe. Parasitol Res. 2014;113: 761-7.

23. Sak B, Kvac M, Hanzlikova D, Cama V. First report of Enterocytozoon bieneusi infection on a pig farm in the Czech Republic. Vet Parasitol. 2008;153:220-4.

24. Zhao W, Zhang W, Yang F, Cao J, Liu H, Yang D, et al. High prevalence of Enterocytozoon bieneusi in asymptomatic pigs and assessment of zoonotic risk at the genotype level. Appl Environ Microbiol. 2014;80:3699-707.

25. Li W, Diao R, Yang J, Xiao L, Lu Y, Li Y, et al. High diversity of humanpathogenic Enterocytozoon bieneusi genotypes in swine in Northeast China. Parasitol Res. 2014;113:1147-53.

26. Wan Q, Lin Y, Mao Y, Yang Y, Li Q, Zhang S, et al. High prevalence and widespread distribution of zoonotic Enterocytozoon bieneusi genotypes in swine in Northeast China: implications for public health. J Eukaryot Microbiol. 2016;63:162-70.

27. Wang SS, Li JQ, Li YH, Wang XW, Fan XC, Liu X, et al. Novel genotypes and multilocus genotypes of Enterocytozoon bieneusi in pigs in northwestern China: a public health concern. Infect Genet Evol. 2018;63:89-94.
28. Del Coco VF, Cordoba MA, Bilbao G, de Almeida CP, Basualdo JA, Santin M. First report of Enterocytozoon bieneusi from dairy cattle in Argentina. Vet Parasitol. 2014;199:112-5.

29. Hu S, Liu Z, Yan F, Zhang Z, Zhang G, Zhang L, et al. Zoonotic and hostadapted genotypes of Cryptosporidium spp., Giardia duodenalis and Enterocytozoon bieneusi in dairy cattle in Hebei and Tianjin, China. Vet Parasitol. 2017:248:68-73.

30. Li J, Luo N, Wang C, Qi M, Cao J, Cui Z, et al. Occurrence, molecular characterization and predominant genotypes of Enterocytozoon bieneusi in dairy cattle in Henan and Ningxia. China Parasit Vectors. 2016;9:142.

31. da Silva Fiuza VR, Lopes CW, de Oliveira FC, Fayer R, Santin M. New findings of Enterocytozoon bieneusi in beef and dairy cattle in Brazil. Vet Parasitol. 2016;216:46-51.

32. Lee $\mathrm{JH}$. Prevalence and molecular characteristics of Enterocytozoon bieneusi in cattle in Korea. Parasitol Res. 2007:101:391-6.

33. Al-Herrawy AZ, Gad MA. Microsporidial spores in fecal samples of some domesticated animals living in Giza. Egypt Iran J Parasitol. 2016;11:195-203.

34. Peng XQ, Tian GR, Ren GJ, Yu ZQ, Lok JB, Zhang LX, et al. Infection rate of Giardia duodenalis, Cryptosporidium spp. and Enterocytozoon bieneusi in cashmere, dairy and meat goats in China. Infect Genet Evol. 2016:41:26-31.

35. Shi K, Li M, Wang X, Li J, Karim MR, Wang R, et al. Molecular survey of Enterocytozoon bieneusi in sheep and goats in China. Parasit Vectors. 2016;9:23.

36. Zhao W, Zhang W, Yang D, Zhang L, Wang R, Liu A. Prevalence of Enterocytozoon bieneusi and genetic diversity of ITS genotypes in sheep and goats in China. Infect Genet Evol. 2015;32:265-70.

37. Li WC, Wang K, Gu YF. Detection and genotyping sudy of Enterocytozoon bieneusi in sheep and goats in east-Central China. Acta Parasitol. 2019;64: 44-50.

38. Lores B, del Aguila C, Arias C. Enterocytozoon bieneusi (microsporidia) in faecal samples from domestic animals from Galicia. Spain Mem Inst Oswaldo Cruz. 2002:97:941-5.

39. Saksirisampant $W$, Prownebon J, Saksirisampant $P$, Mungthin $M$, Siripatanapipong S, Leelayoova S. Intestinal parasitic infections: prevalences in HIV/AIDS patients in a Thai AIDS-care Centre. Ann Trop Med Parasitol. 2009;103:573-81.

40. Chabchoub N, Abdelmalek R, Breton J, Kanoun F, Thellier M, Bouratbine A, et al. Genotype identification of Enterocytozoon bieneusi isolates from stool samples of HIV-infected Tunisian patients. Parasite. 2012;19:147-51.

41. Feng SY, Chang H, Luo J, Huang JJ, He HX. First report of Enterocytozoon bieneusi and Cryptosporidium spp. in peafowl (Pavo cristatus) in China. Int J Parasitol Parasites Wildl. 2019;9:1-6.

42. Abu Samra N, Thompson PN, Jori F, Zhang H, Xiao L. Enterocytozoon bieneusi at the wildlife/livestock interface of the Kruger national park, South Africa. Vet Parasitol. 2012;190:587-90.

43. Karim MR, Dong H, Yu F, Jian F, Zhang L, Wang R, et al. Genetic diversity in Enterocytozoon bieneusi isolates from dogs and cats in China: host specificity and public health implications. J Clin Microbiol. 2014;52:3297-302.

44. Abe N, Kimata I. Molecular survey of Enterocytozoon bieneusi in a Japanese porcine population. Vector Borne Zoonotic Dis. 2010;10:425-7.

45. Wang L, Zhang H, Zhao X, Zhang L, Zhang G, Guo M, et al. Zoonotic Cryptosporidium species and Enterocytozoon bieneusi genotypes in HIVpositive patients on antiretroviral therapy. J Clin Microbiol. 2013;51:557-63.

46. Ye J, Xiao L, Ma J, Guo M, Liu L, Feng Y. Anthroponotic enteric parasites in monkeys in public park. China Emerg Infect Dis. 2012;18:1640-3.

47. Chen D, Wang SS, Zou Y, Li Z, Xie SC, Shi LQ, et al. Prevalence and multilocus genotypes of Enterocytozoon bieneusi in black-boned sheep and goats in Yunnan Province, southwestern China. Infect Genet Evol. 2018:65:385-91.

48. Tamura K, Stecher G, Peterson D, Filipski A, Kumar S. MEGA6: molecular evolutionary genetics analysis version 6.0. Mol Biol Evol. 2013:30:2725-9.

\section{Publisher's Note}

Springer Nature remains neutral with regard to jurisdictional claims in published maps and institutional affiliations. 